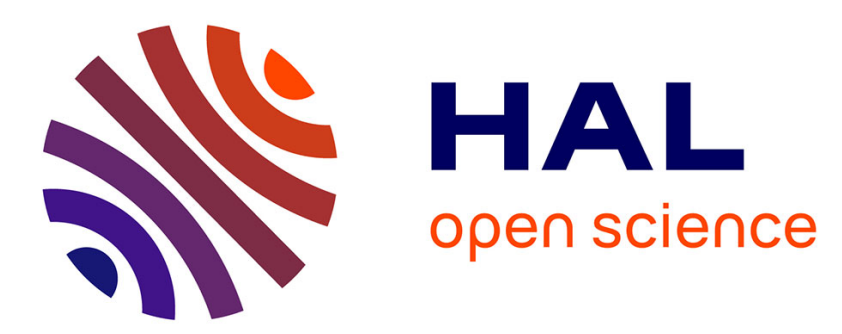

\title{
Pressure of a partially ionized hydrogen gas : numerical results from exact low temperature expansions
}

\author{
Angel Alastuey, Vincent Ballenegger
}

\section{To cite this version:}

Angel Alastuey, Vincent Ballenegger. Pressure of a partially ionized hydrogen gas: numerical results from exact low temperature expansions. Contributions to Plasma Physics, 2010, 50 (1), pp.46-53. 10.1002/ctpp.201010011. hal-00518190

\section{HAL Id: hal-00518190 \\ https://hal.science/hal-00518190}

Submitted on 16 Sep 2010

HAL is a multi-disciplinary open access archive for the deposit and dissemination of scientific research documents, whether they are published or not. The documents may come from teaching and research institutions in France or abroad, or from public or private research centers.
L'archive ouverte pluridisciplinaire HAL, est destinée au dépôt et à la diffusion de documents scientifiques de niveau recherche, publiés ou non, émanant des établissements d'enseignement et de recherche français ou étrangers, des laboratoires publics ou privés. 


\title{
Pressure of a partially ionized hydrogen gas: numerical results from exact low temperature expansions
}

\author{
A. Alastuey*, V. Ballenegger ${ }^{\dagger}$
}

\begin{abstract}
We consider a partially ionized hydrogen gas at low densities, where it reduces almost to an ideal mixture made with hydrogen atoms in their ground-state, ionized protons and ionized electrons. By performing systematic low-temperature expansions within the physical picture, in which the system is described as a quantum electron-proton plasma interacting via the Coulomb potential, exact formulae for the first five leading corrections to the ideal Saha equation of state have been derived [A. Alastuey, V. Ballenegger et al., J. Stat. Phys. 130, 1119 (2008)]. Those corrections account for all effects of interactions and thermal excitations up to order $\exp \left(E_{H} / k T\right)$ included, where $E_{H} \simeq-13.6 \mathrm{eV}$ is the ground state energy of the hydrogen atom. Among the five leading corrections, three are easy to evaluate, while the remaining ones involve suitably truncated internal partition functions of $H_{2}$ molecules and $\mathrm{H}^{-}$and $\mathrm{H}_{2}^{+}$ions, for which no analytical formulae are available in closed form. We estimate those partitions functions at finite temperature via a simple phenomenology based on known values of rotational and vibrational energies. This allows us to compute numerically the leading deviations to the Saha pressure along several isotherms and isochores. Our values are compared with those of the OPAL tables (for pure hydrogen) calculated within the ACTEX method.
\end{abstract}

\section{Introduction}

Obtaining asymptotically exact formulae for the equation of state of quantum Coulomb matter is important, both at a theoretical level and for practical applications. They provide a better understanding of basic phenomena like molecular recombination and screening in the framework of statistical mechanics. Such formulae are free from any a priori phenomenological modelization and uncontrolled approximation. They provide moreover reliable and accurate data in some range of thermodynamical parameters. Exact expansions are of particular interest for hydrogen described as a gas of quantum protons and electrons interacting via the Coulomb potential, since hydrogen is the most abundant element in the universe. A very accurate knowledge of its thermodynamics is needed for example for helioseismology [1, 2, 3]. At low densities, exact expansions have been obtained in two different limits, as described below.

First, in the limit where common proton and electron number density $\rho_{p}=\rho_{e}=\rho$ is driven to zero while temperature $T=1 /\left(k_{B} \beta\right)$ is fixed at a non-zero value, hydrogen becomes fully ionized because of entropy dissociation, as proved in Ref. 活. Then, pressure $P$ can be expanded according to the virial expansion [5, 6, 7, 8,

$$
\beta P=2 \rho-\frac{\kappa^{3}}{24 \pi}+c_{1}(T) \rho^{2}+c_{2}(T) \rho^{5 / 2}+O\left(\rho^{3} \ln \rho\right) .
$$

In that expansion, $\kappa=\left(8 \pi \beta e^{2} \rho\right)^{1 / 2}$ is the familiar Debye wavenumber, while temperature-dependent coefficients $c_{1}(T)$ and $c_{2}(T)$ can be expressed in terms of Ebeling's functions $Q(x)$ and $E(x)$ of a dimensionless parameter $x$ [5]. The leading term describes a classical ideal gas of electrons and protons. The next terms account for corrections due to quantum and interaction effects.

Although the second virial coefficient contains contribution of hydrogen atoms, virial expansion (11) is no longer appropriate in the so-called Saha regime, where a finite fraction of electrons and

\footnotetext{
${ }^{*}$ Laboratoire de Physique, ENS Lyon, CNRS, 46 allée d'Italie, 69364 Lyon Cedex 07, France.

$\dagger$ Institut UTINAM, Université de Franche-Comté, CNRS, 16 route de Gray, 25030 Besançon Cedex, France.
} 
protons recombine into atoms. The second limit of interest precisely defines that Saha regime in a proper mathematical way. According to Saha theory [9], hydrogen is expected to behave as a partially ionized atomic gas at both sufficiently low temperatures and low densities, and its pressure should reduce to Saha formula

$$
\beta P_{\text {Saha }}=\rho+\rho^{*}\left(\sqrt{1+2 \rho / \rho^{*}}-1\right)
$$

with

$$
\rho^{*}=\frac{\exp \left(\beta E_{H}\right)}{2\left(2 \pi \lambda^{2}\right)^{3 / 2}},
$$

$E_{H}=-m e^{4} /\left(2 \hbar^{2}\right)$ the atom groundstate energy, $m$ the reduced electron-proton mass and $\lambda=$ $\left(\beta \hbar^{2} / m\right)^{1 / 2}$. Temperature-dependent density $\rho^{*}$ controls the cross-over between full ionization when $\rho \ll \rho^{*}\left(\beta P_{\text {Saha }} \sim 2 \rho\right)$ and full recombination when $\rho \gg \rho^{*}\left(\beta P_{\text {Saha }} \sim \rho\right)$. That phenomenological prediction has been proved to be true [10] within the scaled double limit $\rho \rightarrow 0$ and $T \rightarrow 0$ while ratio $\rho / \rho^{*}$ is kept fixed, namely pressure $P$ tends to Saha formula (2) discarding exponentially smaller corrections. The corresponding scaled low temperature (SLT) expansion of $P$ reads 11

$$
\beta P / \rho^{*}=\beta P_{S a h a} / \rho^{*}+\sum_{k=1}^{\infty} b_{k}\left(\rho / \rho^{*}\right) \alpha_{k}(\beta) .
$$

Each correction to the leading term $\beta P_{S a h a} / \rho^{*}$ reduces to a function $b_{k}\left(\rho / \rho^{*}\right)$ of ratio $\rho / \rho^{*}$ only, times a temperature-dependent function $\alpha_{k}(\beta)$ which decays exponentially fast when $T$ vanishes, $\alpha_{k}(\beta) \sim \exp \left(-\beta \delta_{k}\right)$ except for possible multiplicative powers of $\beta$. Expansion (4) is ordered with respect to the decaying rates $0<\delta_{1}<\delta_{2}<\ldots$ of the $\alpha_{k}(\beta)$ 's functions. The explicit analytical formulae for the first five corrections obtained in Ref. [11], are recalled in Section 2 , as well as their physical content. As enlighted by its dependence in density $\rho$, each term in SLT expansion (4) is a suitable resummation of an infinite number of terms in virial expansion (11). Such resummations are crucial in order to account non-perturbatively for atomic recombination. Notice that virial coefficients $c_{n}(T)$ diverge in general when $T \rightarrow 0$, like $c_{1}(T)$ which explodes because of the contribution $\exp \left(-\beta E_{H}\right)$ of the atomic groundstate, so virial expansion (11) indeed breaks down in the scaled double limit defining the Saha regime, most terms in the series becoming ill-defined products of the form infinity times zero. Such divergences as $T \rightarrow 0$ also occur in ordinary fugacity expansions in any system where bound states with negative energies exist.

As argued in Refs. 11, 12], the SLT expansion (4) provides an useful analytical knowledge of thermodynamics in an extended domain of the phase diagram. That domain is restricted to sufficiently low temperatures, typically $k_{B} T \ll\left|E_{H}\right|$, and includes of course the fully ionized region $\rho \ll \rho^{*}$ where SLT expansion (14) does reduces to virial expansion (11). Also it includes the atomic region where $\rho$ is of order $\rho^{*}$, and it breaks down at too large densities $\rho \gg \rho^{*}$ because molecular recombination then prevails over atomic recombination. The purpose of the present paper is to give a flavor about reliable numerical data which can be extracted from the truncation of SLT expansion (4) up to order $k=5$ included. Coefficients $b_{k}$ are simple algebraic functions of the reduced density $\rho / \rho^{*}$. If functions $\alpha_{1}(\beta), \alpha_{3}(\beta)$ and $\alpha_{5}(\beta)$ are explicitly known in closed elementary forms, no similar expressions are available for $\alpha_{2}(\beta)$ and $\alpha_{4}(\beta)$, because analytical results on the threeand four-body quantum problem are very scarce. In Section 3, we propose simple modelizations of those functions which account for their exact low-temperature forms one the one hand, and incorporate familiar phenomenological descriptions of ions $\mathrm{H}^{-}$and $\mathrm{H}_{2}^{+}$and of molecule $\mathrm{H}_{2}$ on the other hand. Within those modelizations, we proceed in Section 4 to numerical calculations of the various SLT corrections to Saha EOS (2) along a given isotherm, and we compare our results with those of the OPAL tables [13 calculated within the ACTEX method [14] along a given isochore. More extensive calculations, as well as comparisons with either chemical approaches $[15$ or Path Integral Quantum Monte Carlo simulations [16], will be detailed in a forthcoming paper [17]. 


\section{Exact corrections to Saha pressure}

\subsection{Physical content}

The first five terms in SLT expansion (4) account for non-ideal phenomena such as plasma polarization, shift in the atomic energy levels, interactions between ionized charges and atoms, and also formation of molecules $\mathrm{H}_{2}$ or ions $\mathrm{H}^{-}$and $\mathrm{H}_{2}^{+}$, as summarized in the following table (with obvious notations for groundstate energies of the considered recombined entities).

\begin{tabular}{|c|l|r|}
\hline Correction $(k)$ & Physical content & $\delta_{k}(\mathrm{in} \mathrm{eV})$ \\
\hline 1 & plasma polarization around ionized charges & $\left|E_{H}\right| / 2 \simeq 6.8$ \\
2 & formation of molecules, atom-atom interactions & $\left|3 E_{H}-E_{H_{2}}\right| \simeq 9.1$ \\
3 & atomic excitations, charge-charge interactions & $3\left|E_{H}\right| / 4 \simeq 10.2$ \\
4 & formation of ions, atom-charge interactions & $\left|2 E_{H}-E_{H_{2}^{+}}\right| \simeq 11.0$ \\
5 & fluctuations of plasma polarization & $\left|E_{H}\right| \simeq 13.6$ \\
\hline
\end{tabular}

Further corrections $k \geq 6$ decay exponentially faster than $\exp \left(\beta E_{H}\right)$. The contributions of ion $H^{-}$ are included in the fourth correction, but have decay rate $\left|2 E_{H^{-}}-E_{H^{-}}\right| \simeq 12.9$. The decay rate of the fourth correction is hence determined by the binding energy of ion $\mathrm{H}_{2}^{+}$which is more stable than ion $H^{-}$. Notice that contributions of recombined entities, like atoms $H$ in $k=3$ or molecules $H_{2}$ in $k=2$, are entangled with that of their dissociation products. Moreover, the first correction $k=1$ to Saha pressure is identical to that which can be inferred from a modified Saha ionization equation derived within Green function methods [18. All the other corrections $k \geq 2$ are entirely new, as well as the mathematical structure itself of SLT expansion (4).

\section{$2.2 \quad$ Explicit expressions}

Coefficients $b_{k}$ in SLT expansion (4) are simple algebraic functions of ratio $\rho / \rho^{*}$. The first five coefficients are

$$
\begin{aligned}
& b_{1}\left(\rho / \rho^{*}\right)=\frac{\gamma_{\mathrm{S}}^{3 / 2}\left(\gamma_{\mathrm{S}}-2\right)}{3\left(\gamma_{\mathrm{S}}+1\right)} \\
& b_{2}\left(\rho / \rho^{*}\right)=-\frac{\gamma_{\mathrm{S}}^{4}\left(\gamma_{\mathrm{S}}+3\right)}{2\left(\gamma_{\mathrm{S}}+1\right)} \\
& b_{3}\left(\rho / \rho^{*}\right)=-\frac{\gamma_{\mathrm{S}}^{2}}{\gamma_{\mathrm{S}}+1} \\
& b_{4}\left(\rho / \rho^{*}\right)=-\frac{\gamma_{\mathrm{S}}^{3}\left(\gamma_{\mathrm{S}}+4\right)}{3\left(\gamma_{\mathrm{S}}+1\right)} \\
& b_{5}\left(\rho / \rho^{*}\right)=\frac{\gamma_{\mathrm{S}}^{2}\left(\gamma_{\mathrm{S}}+1-\rho / \rho^{*}\right)}{\left(\gamma_{\mathrm{S}}+1\right)}
\end{aligned}
$$

where $\gamma_{\mathrm{S}}\left(\rho / \rho^{*}\right)=\sqrt{1+2 \rho / \rho^{*}}-1$. For $k=1,2,3,4$, the $\alpha_{k}$ 's functions in expansion (4) are identical to the $h_{k}$ 's functions defined in Ref. [11], while $\alpha_{5}(\beta)=\left[h_{1}(\beta)\right]^{2}$. Function $h_{1}(\beta)$ is given by the simple formula

$$
h_{1}(\beta)=\frac{\left(\beta\left|E_{H}\right|\right)^{3 / 4}}{\pi^{1 / 4}} \exp \left(\beta E_{H} / 2\right)
$$

while

$$
\begin{gathered}
h_{2}(\beta)=\left(\frac{1}{64}\left(\frac{2 m}{M}\right)^{3 / 2} Z(2,2)+W(1,1 \mid 1,1)\right) \exp \left(3 \beta E_{H}\right), \\
h_{3}(\beta)=\left(\frac{1}{2} S_{2}(1,0)+\frac{1}{2} S_{2}(0,1)+W(1,0 \mid 1,0)+W(1,0 \mid 0,1)-\frac{\left(c_{p}+c_{e}\right)\left(\beta\left|E_{H}\right|\right)^{3 / 2}}{24 \pi^{3 / 2}}\right. \\
\left.+\frac{1}{8}\left[Z_{e x c}(1,1)+\left(\frac{2 m}{m_{p}}\right)^{3 / 2} Z(2,0)+\left(\frac{2 m}{m_{e}}\right)^{3 / 2} Z(0,2)\right]\right) \exp \left(\beta E_{H}\right)
\end{gathered}
$$


and

$$
\begin{aligned}
h_{4}(\beta)=\frac{3}{64}\left(\left(\frac{m_{e}\left(M+m_{p}\right)}{M^{2}}\right)^{3 / 2} Z(2,1)+(\right. & \left.\left.\frac{m_{p}\left(M+m_{e}\right)}{M^{2}}\right)^{3 / 2} Z(1,2)\right]+S_{3}(1,1) \\
& \left.+\frac{3}{2}[W(1,1 \mid 1,0)+W(1,1 \mid 0,1)]\right) \exp \left(2 \beta E_{H}\right) .
\end{aligned}
$$

In expressions (11)-(13),$m_{p}$ and $m_{e}$ are respectively the proton and the electron masses, and $M=m_{p}+m_{e}$. Moreover partition functions

$$
Z\left(N_{p}, N_{e}\right)=\frac{\left(2 \pi \lambda_{N_{p}, N_{e}}^{2}\right)^{3 / 2}}{\Lambda} \operatorname{Tr}\left[\exp \left(-\beta H_{N_{p}, N_{e}}\right)\right]^{T}
$$

are truncated traces of the Gibbs operator associated with the Hamiltonian $H_{N_{p}, N_{e}}$ of a cluster of $N_{p}$ protons and $N_{e}$ electrons inside a box with volume $\Lambda$, for which the thermodynamic limit $\Lambda \rightarrow \infty$ is implicitly taken. Also $\lambda_{N_{p}, N_{e}}=\left(\beta \hbar^{2} /\left(N_{p} m_{p}+N_{e} m_{e}\right)\right)^{1 / 2}$ is the thermal de Broglie wavelength of that cluster. Previous truncated traces converge thanks to a truncation inherited from screening effects. Functions $S$ and $W$ describe screening and interaction contributions for the specified clusters.

Functions $\alpha_{1}(\beta)$ and $\alpha_{5}(\beta)$ reduce to elementary functions of $\beta$. Also, two-body partition functions $Z\left(N_{p}, N_{e}\right)$ with $N_{p}+N_{e}=2$ can be rewritten [11] in terms of Ebeling functions $Q(x)$ and $E(x)$ with $x \propto \sqrt{\beta\left|E_{H}\right|}$, which are represented by entire series in $x[6]$. This provides a closed analytical expression for $\alpha_{3}(\beta)$ at finite temperature. Similar expressions for $\alpha_{2}(\beta)$ and $\alpha_{4}(\beta)$ are available only at very low temperatures [11], because of our poor knowledge of the spectra of threeand four-body Hamiltonians $H_{N_{p}, N_{e}}$. Thus, in order to proceed to numerical applications at finite $T$, we first derive simple modelizations of $\alpha_{2}(\beta)=h_{2}(\beta)$ and $\alpha_{4}(\beta)=h_{4}(\beta)$ as described in the next section.

\section{Phenomenological modelizations of some ingredients}

\subsection{Function $h_{2}(\beta)$}

Partition function $Z(2,2)$ defined by (14) involves internal molecular contributions on the one hand, and interaction contributions between entities resulting from molecular dissociation on the other hand. According to the numerical values of $E_{H}, E_{H_{2}^{+}}$, and $E_{H^{-}}$, we can retain only contributions of the molecule and of atom-atom interactions. Indeed, all other contributions decay exponentially faster than $\exp \left(\beta E_{H}\right)$, so they can be dropped for consistency reasons. For the molecular contribution, we use the familiar modelization [19],

$$
\begin{aligned}
& Z_{H_{2}}= \exp \left(-\beta E_{H_{2}}\right) \\
& {\left[\sum_{l=0}^{\infty}(4 l+1) \exp \left(-2 l(2 l+1) \beta \epsilon_{H_{2}}^{(r o t)}\right)+3 \sum_{l=0}^{\infty}(4 l+3) \exp \left(-(2 l+1)(2 l+2) \beta \epsilon_{H_{2}}^{(r o t)}\right)\right] } \\
& {\left[\frac{1}{1-\exp \left(-\beta \epsilon_{H_{2}}^{(v i b)}\right)}\right], }
\end{aligned}
$$

where $\epsilon_{H_{2}}^{(\text {rot })}$ and $\epsilon_{H_{2}}^{(v i b)}$ are the energy quanta associated with global rotation and proton vibration respectively, $\epsilon_{H_{2}}^{(r o t)} / k_{B} \simeq 87.5 K$ and $\epsilon_{H_{2}}^{(v i b)} / k_{B} \simeq 6.34 \times 10^{3} K$. For the contribution of atom-atom interactions, we set

$$
W_{H-H}=\frac{m^{3 / 2}}{8\left(2 \pi \beta \hbar^{2}\right)^{3 / 2}}\left[-\frac{4 \pi}{3} \sigma_{H-H}^{3}+\int_{R>\sigma_{H-H}} \mathrm{~d} \mathbf{R}\left(\exp \left(-\beta U_{H-H}(R)\right)-1\right)\right],
$$

as if the atoms were classical hard spheres with diameter $\sigma_{H-H}$ and interacting via the usual attractive van der Waals potential $U_{H-H}(R)=-A_{H-H} / R^{6}$. In the following, we set $\sigma_{H-H}=3.08 a_{B}$ 
where $a_{B}=\hbar^{2} /\left(m e^{2}\right)$ is the Bohr radius, an oftenly used value for $\sigma_{H-H}$ [6]. Above modelizations of molecular and atomic contributions lead to the simple phenomenological representation

$$
h_{2}^{(\text {phen })}(\beta)=\frac{\sqrt{2} m^{3 / 2}}{32 M^{3 / 2}} Z_{H_{2}} \exp \left(3 \beta E_{H}\right)+W_{H-H} \exp \left(\beta E_{H}\right) .
$$

We stress that modelization (17) does display the exact low-temperature behaviour of $h_{2}(\beta)$, so it should provide rather accurate values for $h_{2}(\beta)$ up to a few thousand Kelvins, where molecular contributions dominate. At very low temperatures, atomic contributions are not well described by $W_{H-H}$ because of quantum effects, but this does not affect significantly the accuracy of $h_{2}^{(p h e n)}(\beta)$. In the range $10000 K<T<30000 K$, atomic contributions become of the same order as molecular contributions. Moreover, large-distance contributions of $U_{H-H}(R)$ to $W_{H-H} \exp \left(\beta E_{H}\right)$ do coincide with the long-range part of the interaction-energy $W(1,1 \mid 1,1) \exp \left(3 \beta E_{H}\right)$ in the exact formula (11). Eventually, contributions of electronic excitations to the internal molecular part $Z_{\mathrm{H}_{2}}$ can be omitted when $T \leq 30000 \mathrm{~K}$, because the corresponding energy gaps are of order $10 \mathrm{eV}$ at least. Therefore, modelization (17) should reasonably work up to $T=30000 \mathrm{~K}$.

\subsection{Function $h_{4}(\beta)$}

Similarly to the above construction of $h_{2}^{(p h e n)}(\beta)$, we introduce

$$
\begin{array}{r}
h_{4}^{(\text {phen })}(\beta)=\frac{3 m_{e}^{3 / 2}\left(M+m_{p}\right)^{3 / 2}}{64 M^{3}} Z_{H_{2}^{+}} \exp \left(2 \beta E_{H}\right)+\frac{3 m_{p}^{3 / 2}\left(M+m_{e}\right)^{3 / 2}}{64 M^{3}} Z_{H^{-}} \exp \left(2 \beta E_{H}\right) \\
+\frac{c_{a t}}{8 \pi^{3 / 2}\left(\beta\left|E_{H}\right|\right)^{1 / 2}} \exp \left(2 \beta E_{H}\right)+W_{H-p} \exp \left(\beta E_{H}\right)+W_{H-e} \exp \left(\beta E_{H}\right) .
\end{array}
$$

In such modelization, internal partition functions for ions $\mathrm{H}_{2}^{+}$and $\mathrm{H}^{-}$are defined as

$$
\begin{aligned}
& Z_{H_{2}^{+}}=2 \exp \left(-\beta E_{H_{2}^{+}}\right) \\
& {\left[\sum_{l=0}^{\infty}(4 l+1) \exp \left(-2 l(2 l+1) \beta \epsilon_{H_{2}^{+}}^{(r o t)}\right)+3 \sum_{l=0}^{\infty}(4 l+3) \exp \left(-(2 l+1)(2 l+2) \beta \epsilon_{H_{2}^{+}}^{(r o t)}\right)\right] } \\
& {\left[\frac{1}{1-\exp \left(-\beta \epsilon_{H_{2}^{+}}^{(v i b)}\right)}\right] }
\end{aligned}
$$

and

$$
Z_{H^{-}}=2 \exp \left(-\beta E_{H^{-}}\right),
$$

with $\epsilon_{H_{2}^{+}}^{(r o t)} / k_{B} \simeq 43.1 K$ and $\epsilon_{H_{2}^{+}}^{(v i b)} / k_{B} \simeq 3.34 \times 10^{3} K$, and where electronic excitations are omitted. Contribution of $S_{3}(1,1) \exp \left(2 \beta E_{H}\right)$ has been replaced by its low-temperature form with constant $c_{a t}$ given in Ref. [11]. Contributions of interactions between an atom and an ionized charge are expressed as respectively

$$
W_{H-p}=\frac{3 m^{3 / 2}}{4\left(2 \pi \beta \hbar^{2}\right)^{3 / 2}}\left[-\frac{4 \pi}{3} \sigma_{H-p}^{3}+\int_{R>\sigma_{H-p}} \mathrm{~d} \mathbf{R}\left(\exp \left(-\beta U_{H-p}(R)\right)-1\right)\right]
$$

and

$$
W_{H-e}=\frac{3 m^{3 / 2}}{4\left(2 \pi \beta \hbar^{2}\right)^{3 / 2}}\left[-\frac{4 \pi}{3} \sigma_{H-e}^{3}+\int_{R>\sigma_{H-e}} \mathrm{~d} \mathbf{R}\left(\exp \left(-\beta U_{H-e}(R)\right)-1\right)\right],
$$

where we set $\sigma_{H-p}=2.54 a_{B}$ and $\sigma_{H-e}=1.54 a_{B}$. Furthermore, $U_{H-p}(R)=-A_{H-p} / R^{4}$ and $U_{H-e}(R)=-A_{H-e} / R^{4}$ are the usual potentials generated by quantum fluctuations of dipolecharge interactions.

Similarly to modelization (17), phenomenological expression (18) reproduces the exact leading behaviour of $h_{4}(\beta)$ at low temperatures, while large-distance contributions of $U_{H-p}(R)$ and $U_{H-e}(R)$ 
to $W_{H-p}$ and $W_{H-e}$ respectively, reduce to their counterparts in exact formula (13). That expression cannot be expected to be very accurate up to $T=30000 \mathrm{~K}$, because of various drawbacks, like the occurence of quantum effects for the ionized electron. Nonetheless, the corresponding accuracy is sufficient for our purpose, because contributions to thermodynamics associated with $h_{4}(\beta)$ remain quite small in the Saha regime.

\section{Numerical results}

\subsection{Low-temperature isotherm}

In Fig. 1, we plot deviation $\delta P=P-P_{\text {Saha }}$ computed by retaining the first five correction terms in SLT expansion (何), along isotherm $T=6000 \mathrm{~K}$ for which $\rho^{*} \simeq 2.16 \times 10^{15} \mathrm{~m}^{-3}$, as a function of reduced density $\rho / \rho^{*}$. When $\rho / \rho^{*} \leq 10^{5}$, the dominant contribution is due to the polarization of the plasma around ionized charges, embedded in correction $k=1$. For $\rho / \rho^{*} \leq 10^{-2}$, that contribution is negative and reduces to the Debye expression $-\kappa^{3} /(24 \pi)$, in agreement with virial expansion (11). At $\rho / \rho^{*}=4$, correction $k=1$ changes sign, as seen on expression (5) for coefficient $b_{1}\left(\rho / \rho^{*}\right)$. The plasma-polarization correction is thus not given at high densities by Debye formula with a modified Debye length computed with the density of ionized charges, as it could naively be expected.

At densities $\rho / \rho^{*} \geq 10^{5}$, molecular contributions embedded in term $k=2$ become the most important correction, as expected at high densities. Since the formation of molecules reduces the pressure, $\delta P$ then becomes again negative. When $\rho / \rho^{*} \geq 10^{11}$, the SLT expansion fails to converge because molecular recombination cannot be treated perturbatively anymore. Notice that corrections $k=3,4,5$ give negligible contributions along the present isotherm. This is no longer the case at higher temperatures, where term $k=3$, which involves atomic excitations and atomatom interactions, overcomes the other corrections in some density range, as exemplified along the isochore shown in the next section.

Eventually, let us illustrate above considerations through an astrophysical example of wide interest. In the Sun photosphere, the temperature is indeed about $6,000 \mathrm{~K}$. If we assume a massic density of order $10^{-7} \mathrm{~g} / \mathrm{cc}$ and relative concentrations in mass of $72 \%$ Hydrogen, $26 \%$ Helium and $2 \%$ for heavier elements, we find $\rho / \rho^{*} \simeq 10^{7}$ [20]. Under such conditions, electrons and protons are almost fully recombined into hydrogen atoms, and the deviations to Saha pressure are primarily due to the formation of $\mathrm{H}_{2}$ molecules.

\subsection{Low-density isochore and comparison to OPAL equation of state}

Now we consider an isochore along a fixed density $\rho$ corresponding to a massic density equal to $10^{-7} \mathrm{~g} / \mathrm{cc}$. In Fig. 2, we compare deviations to ideal Saha pressure obtained by keeping the first five terms in SLT expansion (4), with the tabulated values of the OPAL equation of state [13]. Because of the presence of exponential factors, the results are very sensitive to the used value for the atomic groundstate energy, in particular when determining $\delta P_{\mathrm{OPAL}}=P_{\mathrm{OPAL}}-P_{\text {Saha }}$. After checking OPAL data in some specific limits where all corrections to ideal Saha pressure can be safely neglected, we conclude that we have to compute $\delta P_{\text {OPAL }}$ with ideal Saha pressure (2) determined with the Rydberg energy $E_{\mathrm{Ryd}} \simeq-13.6057 \mathrm{eV}$ instead of the true value $E_{H}=-m e^{4} /\left(2 \hbar^{2}\right) \simeq$ $-13.598286 \mathrm{eV}$. Indeed, that difference caused by the reduced mass can induce corrections of more than one order of magnitude in $\delta P$ in regions where $\rho \sim \rho^{*}$.

At low temperatures $T<8000 \mathrm{~K}$, the dominant correction along the isochore is due to molecular recombination embedded in term $k=2$. Our data agree relatively well with the OPAL values. At very low temperatures, $T<3000 \mathrm{~K}$, expansion (4) cannot be used at the considered density, because molecular recombination becomes then too important.

When $T>8000 \mathrm{~K}$, the OPAL values are clearly very well reproduced, by the sole correction $k=1$. For $T>11000 \mathrm{~K}$, contributions of charge-charge interactions and of atomic excitations 


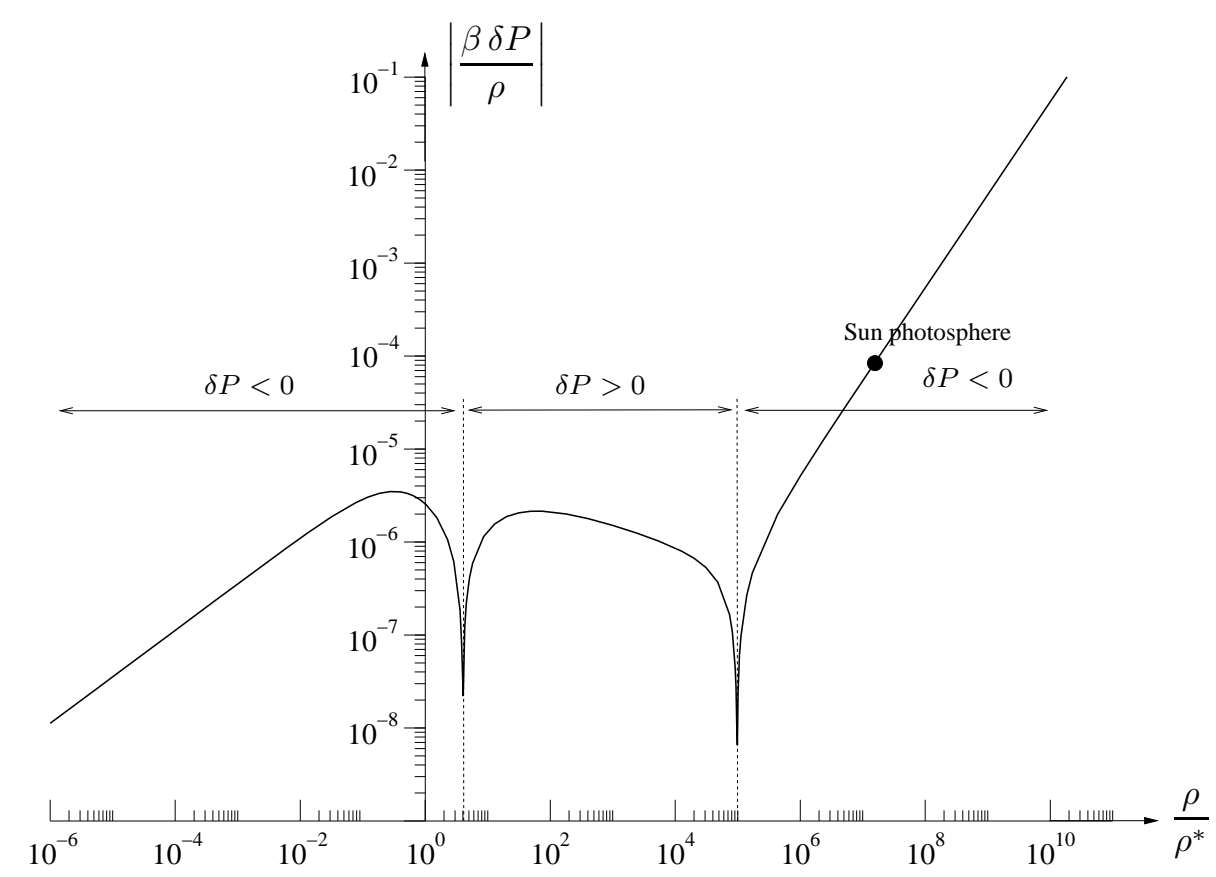

Figure 1: Logarithmic plot of deviations to Saha pressure for pure hydrogen along isotherm $T=$ $6,000 \mathrm{~K}\left(\rho^{*}=2.1 \times 10^{15} \mathrm{~m}^{-3}\right)$.

embedded in correction $k=3$, overcome term $k=1$. More extensive calculations and further comparisons will be detailed in a forthcoming publication [17]. Also, we will present results for other thermodynamical quantities (in particular the internal energy) which can be obtained using standard thermodynamical relations.

\section{References}

[1] J. Christensen-Dalsgaard, W. Däppen et al., The current state of solar modeling, Science 272: 1286-1292

[2] W. Däppen, The equation of state for the solar interior, J. Phys. A: Math. Gen. 39: 4441 (2006)

[3] A. N. Starostin and V. Roerich, Bound states in nonideal plasmas: formulation of the partition function and application to the solar interior, Plasma Sources Sci. Technol. 15 (2006):410-415

[4] J. Lebowitz and R. Pena, Low density form of the free energy of real matter, J. Chem. Phys. 59:1362-1364 (1973)

[5] W. Ebeling, Ann. Phys. Leipz. 19:104 (1967)

[6] W.D. Kraeft, D. Kremp, W. Ebeling, and G. Ropke, Quantum Statistics of Charged Particle Systems (Plenum Press, New York, 1986)

[7] A. Alastuey and A. Perez, Virial expansion of the equation of state of a quantum plasma, Europhys. Lett. 20 :19-24 (1992)

[8] T. Kahlbaum, The quantum-diffraction term in the free energy for Coulomb plasma and the effective-potential approach, J. Phys. IV 10 (P5):455 (2000)

[9] M. Saha, Philos. Mag. 40:472 (1920)

[10] N. Macris and Ph.A. Martin, Ionization equilibrium in the proton-electron gas, J. Stat. Phys. 60:619-637 (1990)

[11] A. Alastuey, V. Ballenegger, F. Cornu and Ph.A. Martin, Exact results for thermodynamics of the hydrogen plasma: low-temperature expansions beyond Saha theory, J. Stat. Phys. 130:1119-1176 (2008)

[12] A. Alastuey and V. Ballenegger, J. Phys. A: Math. Theor. 42 (2009): 214031 

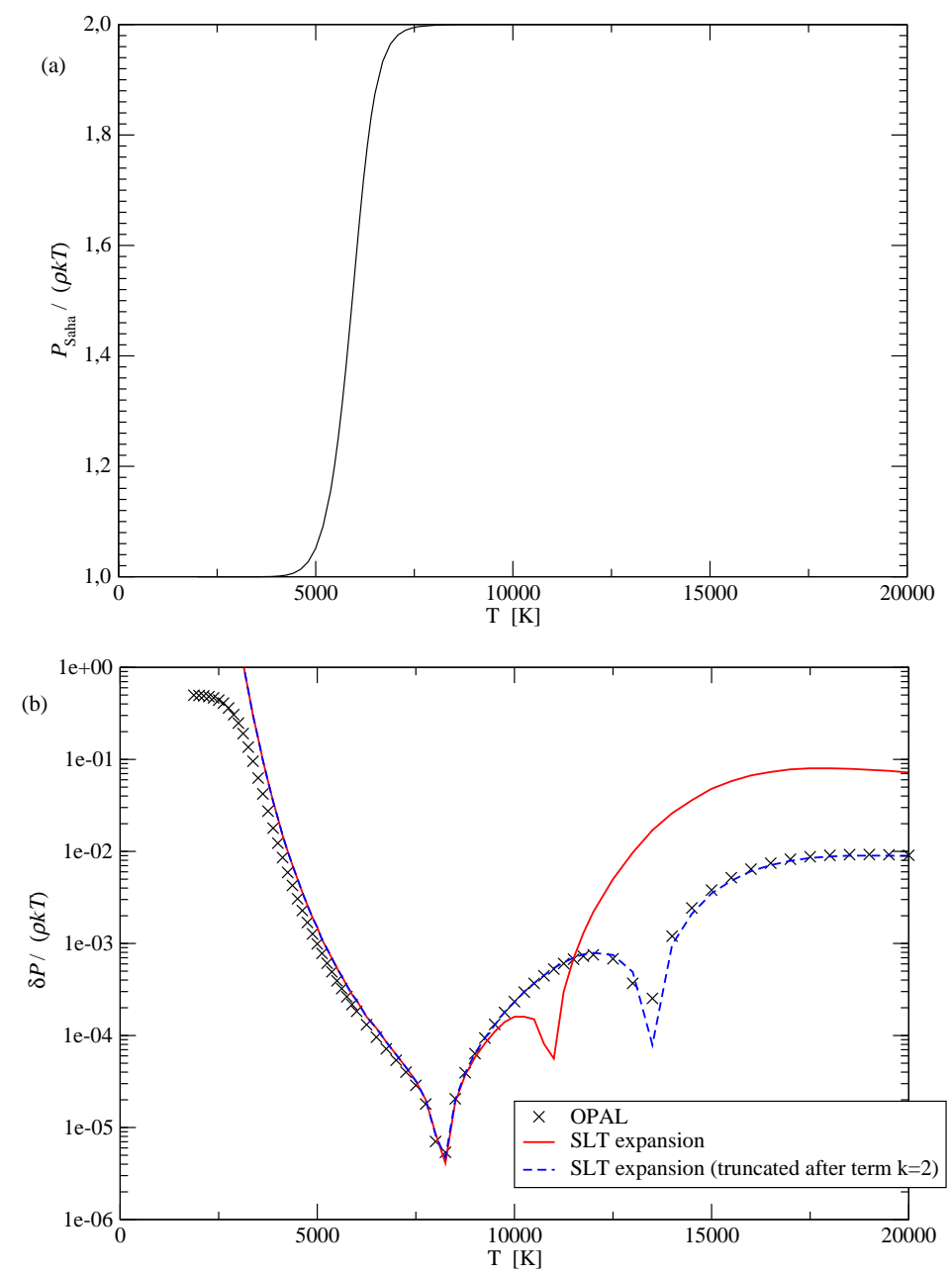

Figure 2: Pressure along isochore $10^{-7} \mathrm{~g} / \mathrm{cc}$ : (a) ideal Saha pressure [eq. (2)]; (b) Logarithmic plot of deviations to ideal Saha pressure, calculated using the SLT expansion of pressure truncated after term $k=5$ (solid line) and $k=2$ (dashed line). Crosses correspond to the tabulated OPAL equation of state [13].

[13] F.J. Rogers and A. Nayfonov, ApJ 576:1064-1074; http://www.phys.llnl.gov/Research/OPAL/index.html

[14] F.J. Rogers, Statistical mechanics of Coulomb gases of arbitrary charge, Phys. Review A 10: $2441(1974)$

[15] V K Gryaznov, S V Ayukov et al., Solar plasma: calculation of thermodynamic functions and equation of state, J. Phys. A: Math. Gen. 39 (2006): 4459-4464

[16] B. Militzer and D.M. Ceperley, Path integral Monte Carlo simulation of the low-density hydrogen plasma, Phys. Rev. E 63:066404 (2001)

[17] A. Alastuey, V. Ballenegger and F. Cornu, in preparation

[18] D. Kremp, W.D. Kraeft and A.J.M.D. Lambert, Equation of state and ionization equilibrium for non-ideal plasmas, Physica A 127:72-86 (1984)

[19] L.D. Landau and E.M. Lifchitz, Quantum mechanics, vol. 3 of the course of Theoretical Physics, 3rd edition (Pergamon Press, Oxford, 1977)

[20] J. A. Eddy, New Sun: The Solar Results From Skylab (NASA SP-402, edited by R. Ise, Washington D.C., 1979) 